\title{
Diabetes Control and Hypoglycemia
}

\author{
Paul Norwood and Alex Fogel \\ Valley Endocrine Research, Fresno, CA \\ University of California, San Francisco
}

USA

\section{Introduction}

Diabetes management has traditionally focused solely on lowering blood glucose levels to avoid the long term complications of diabetes. This approach sometimes neglects the detrimental effects of hypoglycemia on the diabetic patient. This is problematic, as hypoglycemia often places patients at risk of poor quality of life, increased medical costs and significant complications. As many instances of hypoglycemia are preventable by smarter treatment strategies, it is expected that accounting for the possibility of hypoglycemia may not only reduce a patient's health care costs, but will allow him or her a better quality of life, decrease the potential for disability and even increase life-expectancy. This article provides an overview of hypoglycemia and establishes guidelines for the management and prevention of hypoglycemia based on the most current research.

\section{Definitions}

Hypoglycemia is defined as a state in which there are neuroglycopenic symptoms concurrent with a low blood glucose level. The definition of "low blood glucose" can differ significantly across the major medical associations. While the American Diabetes Association (ADA) defines low blood glucose as glucose levels less than or equal to 70 $\mathrm{mg} / \mathrm{dL}$ (3.9 mM) (ADA, 2005), the European Medicines Agency (EMA) defines the condition as glucose levels under $54 \mathrm{mg} / \mathrm{dL}(3.0 \mathrm{mM})$ (EMA, 2010). The ADA definition of "low blood glucose" will be used for the purposes of this article.

It should be noted that despite this baseline threshold, the symptoms of hypoglycemia present at different levels in different patients. For example, in patients averaging blood glucose levels above $200 \mathrm{mg} / \mathrm{dL}$, neurogenic symptoms associated with hypoglycemia can appear when the blood glucose falls to what should be a normal level, near or below 100 $\mathrm{mg} / \mathrm{dL}$. This not only presents a significant difficulty in preventing hypoglycemic symptoms, but the glucose situation of each patient must be considered individually as well.

\subsection{Artifactual hypoglycemia}

In determining whether a patient is experiencing hypoglycemia, it is essential to recognize the situations that can frequently result in false-positives. Artifactual hypoglycemia can be the product of a number of factors, including some that are a result of the methodology employed. These include the absence of an antiglycolytic agent in the blood collection tube, 
delayed blood processing, and imperfect glucose monitoring machines, which are often accurate only to within $+/-40 \mathrm{mg} / \mathrm{dL}$. In this last example, a patient meeting both the EMA and the ADA criteria for hypoglycemia may actually be in the normal blood glucose range. Patient health factors that affect the true detection of hypoglycemia are typically those that result in an abnormal consumption of blood glucose by circulating cells. In patients with leukemia, elevated levels of glycolysis in leukocytes result in significantly decreased levels of blood glucose from the time blood is drawn to the time blood is monitored (Spiller, 2011). The effect is similar in patients suffering from erythrocytosis and polycythemia vera.

\section{Symptoms of hypoglycemia}

The symptoms of hypoglycemia can be divided into two distinct categories: neuroglycopenic and neurogenic. In both types of diabetes, neuroglycopenic and neurogenic hypoglycemic symptoms can appear with a large change in blood glucose levels without achieving actual numerical hypoglycemia.

\subsection{Neuroglycopenic symptoms}

The neuroglycopenic symptoms of hypoglycemia include (Ben-Ami et al., 2009; Warren, 2005):

- Behavioral changes, including irritability and nightmares

- Cognitive impairments, including confusion and difficulty concentrating

- Psychomotor abnormalities, including dizziness, weakness, and poor coordination

- Seizure

- Coma

\subsection{Neurogenic symptoms}

The neurogenic symptoms of hypoglycemia include (Warren, 2005):

- $\quad$ Adrenergic tremor

- Palpitations and anxiety

- Colinergic sweating

- Hunger

- Paresthesias or neurological symptoms, such as numbness in the mouth and tongue

\section{Causes of hypoglycemia in diabetic patients}

In patients with either type I or type II diabetes, the root cause of factual hypoglycemia is always hyperinsulinemia. However, the etiology of hyperinsulinemia varies depending on the type of treatment strategy in place. For type I diabetes, hypoglycemia is always due to excessive insulin dosage. In type II diabetes, it results from the use of insulin or sulfonureas. The combination of a GLP-1 agent and a sulfonurea is a potent mixture and may cause hypoglycemia.

The risk of hypoglycemia in patients with type II diabetes beginning insulin treatment is fairly low, and is comparable to patient populations being treated with sulfonureas alone. However with time the incidence increases remarkably. For type II diabetics, the incidence increases from $5 \%$ for patients on insulin for less than two years to $24 \%$ for type II patients taking insulin for more than five years (Heller, 2010). In type I diabetics, the incidence of 
hypoglycemia increases from $22 \%$ in patients with the condition for less than 5 years to $44 \%$ in patients who have had diabetes for more than 15 years.

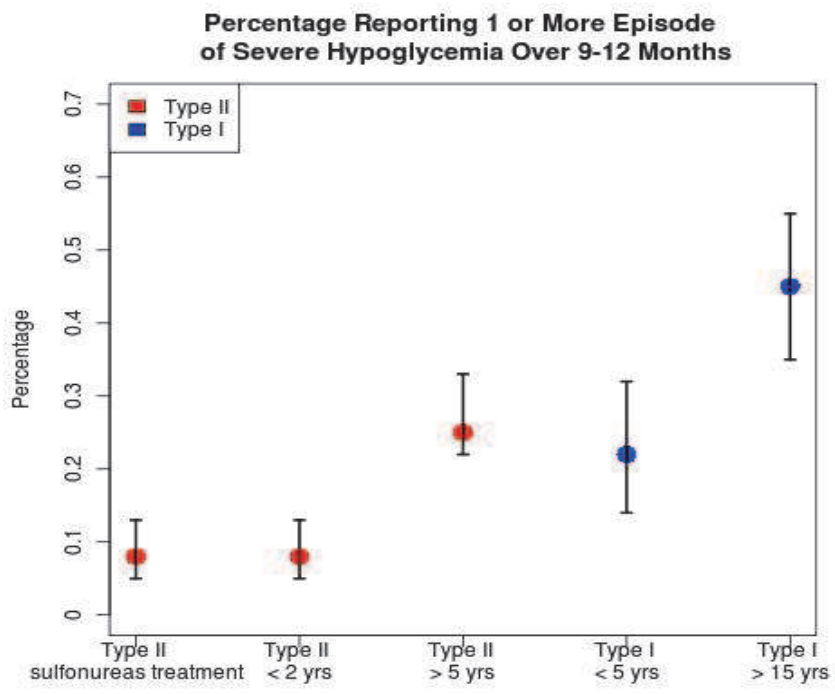

Fig. 1. Incidence of hypoglycemia increases with the duration of illness. (adapted from Heller, 2010).

The increase of hypoglycemia likely results from impaired counter-regulatory hormone release (Bolli, 1983; Matyka, 1997). Within five years of the onset of type I diabetes, expression of the hypoglycemia-protective hormone glucagon is less responsive to instances of hypoglycemia by $66 \%$. This imbalance is partially mitigated by normal epinephrine levels during this time. However, by fifteen years of the disease, type I diabetics typically present with glucagon levels decreased by $80 \%$ of baseline and epinephrine levels reduced by $66 \%$ (Heller, 2010). This situation presents a significant difficultly for the patient's counterregulatory hormones to correct. Type II diabetics also develop the same hormonal deficiencies, which are proportional to the duration of the disease.

In both type I and type II diabetes patients, the situation is enhanced by physiological unawareness of hypoglycemia. Hypoglycemic unawareness occurs in $17 \%$ of patients who have had type I diabetes (Heller, 2010). The causes are:

- Having diabetes for many years with decreased counter-regulatory hormones

- Autonomic neuropathy

- Frequent low blood sugar with desensitization to hypoglycemia

- Stress or depression

- Poor self-care

- Alcohol consumption

- A previous low blood sugar in the last 24 to 48 hours

- Beta blockers 


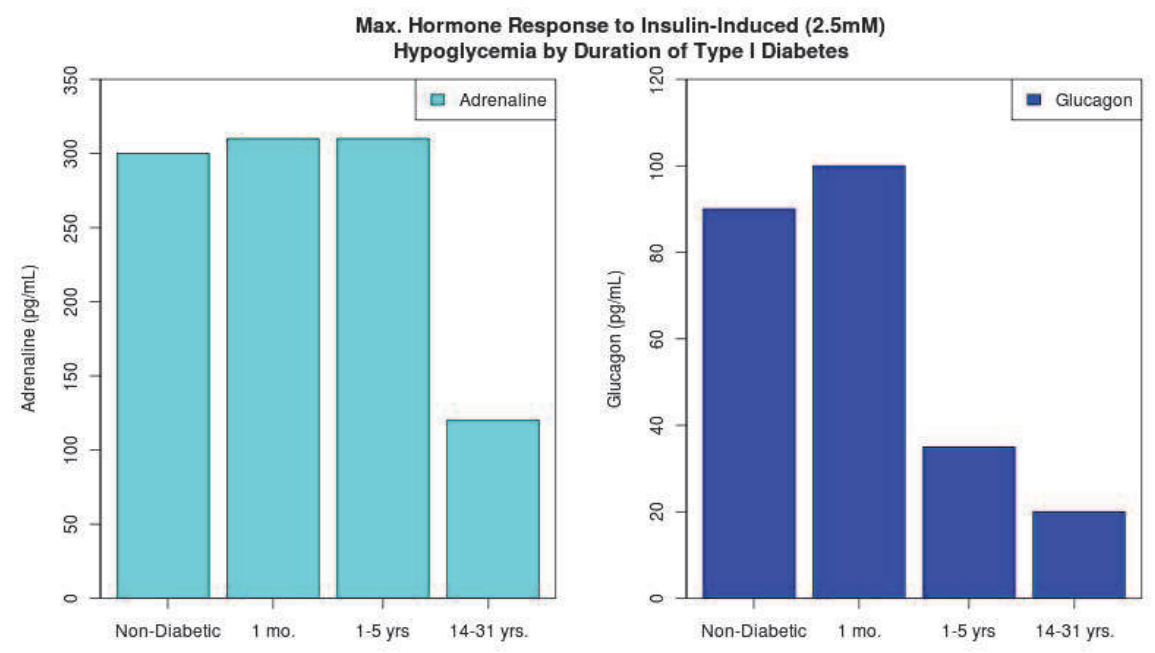

Fig. 2. Progressive decrease in endocrine counter-regulation depending on duration of Type I diabetes. (adapted from Heller, 2010).

\subsection{Normal physiological response to of hypoglycemia}

As the brain cannot synthesize glucose or store substantial amounts as glycogen in neurons, it requires a continuous supply of glucose from the circulation to function. Hypoglycemia therefore causes brain dysfunction. The normal physiologic response to hypoglycemia appears in stages. Epinephrine release begins at $63 \mathrm{mg} / \mathrm{dL}$ (Cryer, 2007). Secondary symptoms of tremor, palpitations and sweating begin at $58 \mathrm{mg} / \mathrm{dL}$. Cognitive dysfunction presents consistently at $54 \mathrm{mg} / \mathrm{dL}$. Confusion and disorientation appear at $45 \mathrm{mg} / \mathrm{dL}$. At 18 $\mathrm{mg} / \mathrm{dL}$, coma and seizure result. Prolonged blood glucose levels less than $18 \mathrm{mg} / \mathrm{dL}$ result in brain damage.

\subsection{Impaired physiological responsiveness}

In diabetics exhibiting impaired physiological responsiveness to hypoglycemia and hypoglycemic unawareness, the levels of blood glucose corresponding to the stages of cognitive dysfunction are unchanged. However, the release of epinephrine occurs at 50-52 $\mathrm{mg} / \mathrm{dL}$, which is lower by $10 \mathrm{mg} / \mathrm{dL}$ than the typical release point observed in normal patients. It should be noted that epinephrine release does not occur until after cognitive dysfunction appears. Thus, the warning symptoms of tremor, palpitations and sweating actually follow cognitive dysfunction instead of preceding it. This type of brain desensitization to hypoglycemia is caused by acclimation to frequent episodes of hypoglycemia. The brain becomes accustomed to the low glucose levels and does not signal for epinephrine to be released during such times. Because of neuroglycopenia, the patient with an impaired epinephrine response cannot reliably treat his or her own condition. In these cases, the only hopes for the patient to combat the episode of hypoglycemia are natural insulin degradation, delayed epinephrine and glucagon release, or intervention by a third party. 


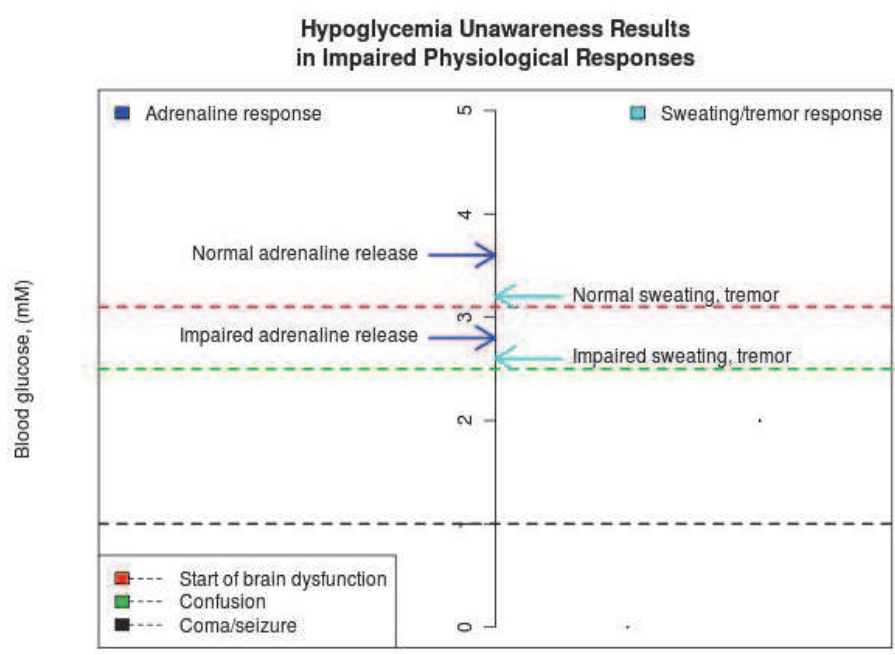

Fig. 3. Impaired physiological responses due to hypoglycemia unawareness (adapted from Cryer, 2007). Note: $1 \mathrm{mM}=18 \mathrm{mg} / \mathrm{dL}$

The reason for this reduced responsiveness is the brain's adaptation to frequent episodes of hypoglycemia. In patients experiencing frequent bouts of hypoglycemia, glucose transporters in the neurons of the brain increase in number, permitting the brain to receive a more steady supply of glucose. As a result, the blood hypoglycemic threshold for the brain to signal epinephrine release becomes lower. This creates a state of hypoglycemic unawareness, as the brain has adapted to hypoglycemia. The patient can then develop perilously low glucose levels without realizing it.

Since repeated hypoglycemia is common in people with diabetes who strive to keep their glucose levels under tight control near a pre-defined 'normal' level, the incidence of hypoglycemic unawareness is higher in this population. The more intensive the treatment protocol, the greater the incidence of hypoglycemia and hypoglycemia unawareness. This situation is abetted by the rigidity of the patient's diabetes control, as many patients will ignore and attempt to tolerate their hypoglycemic episodes to meet the specifications of their physician. This population is at serious risk of becoming accustomed to hypoglycemia, and unfortunately, these patients run a risk of developing a major hypoglycemic reaction and the associated medical consequences. To successfully monitor this situation, the physician must realize that some patients experience difficulty in loosening their tight blood glucose control, and these patients should be warned repeatedly about the dangers of hypoglycemic episodes and its consequences.

In addition, stress, depression, poor self-care, alcohol or drug use or any situation that renders the patient less attentive or aware of his or her bodily sensations can contribute to hypoglycemic unawareness. In particular, depression, which is found in approximately $30 \%$ of diabetics, needs to be addressed (ADA, 2005; Piette et al., 2004). It is very difficult for patients suffering from clinical depression to control their diabetes. As a physician, one must be alert for classic depressive symptoms in their diabetic patients, principally fatigue. It takes energy and effort to care for diabetes, and if the patient is depressed, he or she will experience great difficulty in performing simple self-care. Many of these patients lack the 
energy to monitor their diabetes, the self-motivation to adhere to a diet, and the focus to coordinate to two. This frequently leads to hypoglycemic reactions.

Beta blockers can also contribute to the incidence of hypoglycemia. These drugs decrease the beta adrenergic symptoms, such as palpitations and tremor, that are associated with the onset of hypoglycemia. On occasion, these medications may prevent the patient from recognizing the adrenergic warning symptoms, resulting in hypoglycemic unawareness. Beta blockers also prevent epinephrine from increasing the release of glucose from the liver.

\section{Prevalence of hypoglycemia}

The prevalence of hypoglycemia is profound and worrisome, especially considering the lack of attention given to the condition. In one study in which patients self-reported hypoglycemic events, $50 \%$ of patients with type II diabetes on insulin and $30 \%$ of patients taking sulfonureas without insulin reported such an event (Cryer, 2003). It has been shown that $62 \%$ of type I diabetics exhibit asymptomatic hypoglycemia, as do $46 \%$ of type II diabetics. There is also evidence that type I diabetics can expect to experience a major hypoglycemic event at least once per year, while type II diabetics can anticipate a similar event once every 2.5 years. As the prevalence of type II diabetes is far greater than type I diabetes, the absolute number of hypoglycemic events in type II diabetes is significantly higher than in type I diabetics, but the incidence per capita is lower.

\subsection{Determinants of hypoglycemic events}

Several determinants can be used to predict an increased risk of symptomatic hypoglycemia. These include:

- Advanced age

- Irregular meal schedule

- Regularity of exercise

- Hemoglobin A1c $\geq 7 \%$

- Having diabetes $>8$ years

- Duration of insulin therapy

- Renal impairment

- Diabetic neuropathy

- Previous history of hypoglycemia

- Anticoagulant therapy

- Concurrent medication of five or more prescription drugs hospitalization within the last 12 months

The state of the patient's health is therefore a indicator of the incidence of significant hypoglycemia. The more fragile the patient, the more likely he or she is to experience a severe hypoglycemic event.

\section{Hypoglycemia and increased mortality}

There are two potential causative reasons for increased mortality in patients with hypoglycemia. The main one is cardiovascular. Diabetics have a higher incidence of cardiovascular disease than the general population, including coronary artery disease, cardiac hypertrophy, and cardiac autonomic neuropathy (Johnston, 2011). This is because 
with hypoglycemia, there is an increase adrenergic load causing a faster heart rate with subsequent cardiac ischemia and possibly myocardial infarction. Hypoglycemia also causes a increased QT interval on the electrocardiogram. The QT interval is increased more in those with diabetic autonomic neuropathy. It is well documented that an increased QT interval can lead to ventricular fibrillation and sudden death (Lee, 2004).

\section{Normal EKG}

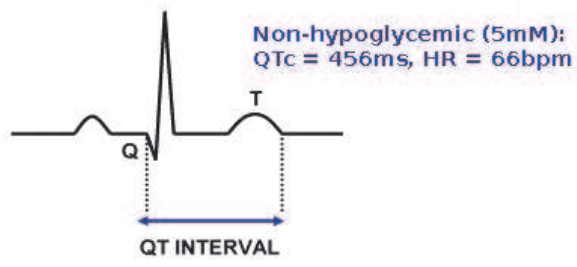

\section{Prolonged QT EKG}

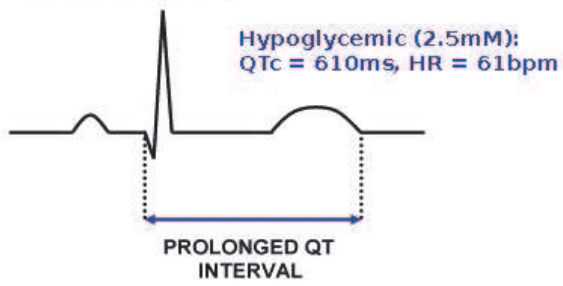

Fig. 4. QT interval prolongation during hypoglycemia (adapted from Heller 2010).

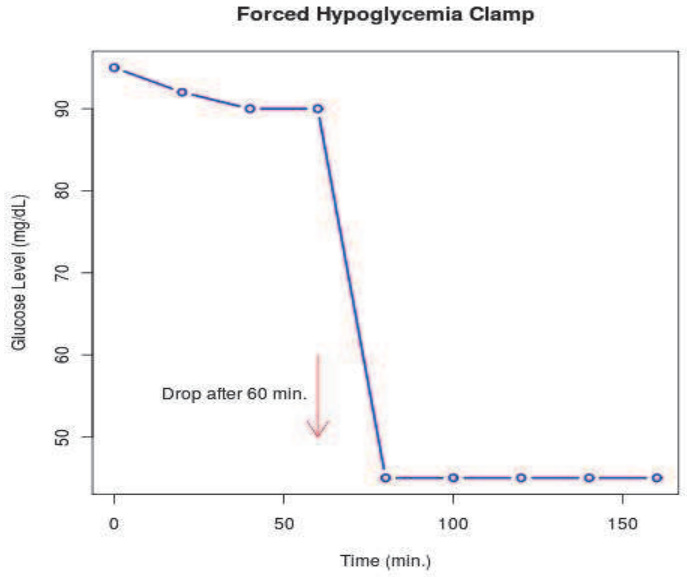

Fig. 5. Drop in glucose after 60 minutes with a insulin-glucose clamp. Glucose lowered with insulin from $95 \mathrm{mg} / \mathrm{dL}$ to $45 \mathrm{mg} / \mathrm{dL}$ (adapted from Lee, 2004). Please refer to Figure 6 to connect the drop in blood glucose with prolonged QTc interval. 


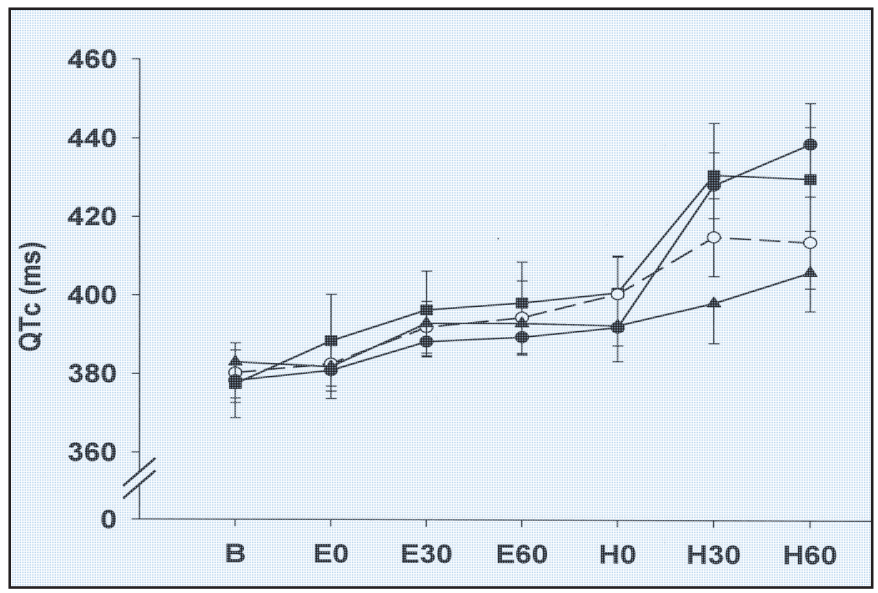

Fig. 6. The effect of noted hypoglycemia on QTc interval in ms. Mean \pm SE QTc at baseline (B) after 0, 30, and $60 \mathrm{~min}$ of euglycemia (E0, E30, and E60, respectively) and hypoglycemia (H0, H30, and H60, respectively) (adapted from Lee, 2004).

\subsection{Hypoglycemia and the brain}

As glucose is necessary for the brain's metabolic function, frequent severe hypoglycemia results in an increased risk of brain cell death, and has been linked to an increased incidence of dementia (Bruce, 2009).

However, the Fremantle study presents a strong counter argument to this hypothesis, finding that elderly patients with hypoglycemia do not have persistent adverse neurological effects (Fremantle Diabetes Study, 2009). It should be noted that elderly patients with dementia are highly susceptible to hypoglycemia. This group hypothesized that the well recognized brain damage associated with repeated severe hypoglycemia is more likely in incidences in which symptom recognition and the ability to self-correct or seek assistance are impaired, for example in patients with cognitive impairment or dementia. Hypoglycemia may be the cause of death in type I and type II diabetics and is found in those who have severe secondary complications of diabetes, such as end stage renal failure or severe autonomic neuropathy.

However, hypoglycemia is a part of life for many diabetics. Some patients may be hypoglycemic, with blood glucose less than $50 \mathrm{mg} / \mathrm{dL}$, up to $10 \%$ of the time (Cryer, 2007). As previously noted, type I diabetics can statistically expect to experience at least one major hypoglycemic reaction per year. Thankfully, death is rare in these instances. In primate studies, it takes 5-6 hours of hypoglycemia (blood glucose less than $20 \mathrm{mg} / \mathrm{dL}$ with an average of $13 \mathrm{mg} / \mathrm{dL}$ ) to produce brain damage and brain death (Cryer, 2007). Thus, it is extremely difficult to cause brain death by isolated instances of hypoglycemia in diabetics who have good hormonal protective mechanisms and who do not have major complications. Brain dysfunction caused by temporal hypoglycemia, a condition that many clinicians treat daily, is usually reversed quickly with the reintroduction of glucose. Fully $70 \%$ of major hypoglycemic reactions which necessitate professional assistance are treated in outpatient facilities. In fact, a small benefit of recurrent moderate hypoglycemia is that the increase in neuronal glucose uptake transporters may actually ameliorate brain damage and 
cognitive dysfunction induced by severe instances of hypoglycemia, as the brain is able to obtain more glucose than would be expected in a naïve patient.

\section{Recent hypoglycemia trial results (ACCORD, ADVANCE, VADT, NICE- SUGAR)}

Hypoglycemia was found to be a major health issue for diabetics in four recent large-scale studies: the Action to Control Cardiovascular Risk in Diabetes Trial (ACCORD), the Action in Diabetes and Vascular Disease: Controlled Evaluation (ADVANCE), VA Diabetes Trial (VADT) and the Normoglycaemia in Intensive Care Evaluation and Survival Using Glucose Algorithm Regulation Trial (NICE-SUGAR). (NICE-SUGAR Study, 2009).

Each of the ACCORD, ADVANCE and VADT studies examined intensively treated diabetics as outpatients, and compared this population with standard treatments, with the major endpoints looking at CV death, non-fatal myocardial infarctions and strokes. Other macrovascular events were looked at in the ADVANCE and VADT.

\begin{tabular}{|c|c|c|c|}
\hline \multicolumn{4}{|c|}{ Study Designs: ACCORD, ADVANCE, VADT } \\
\hline & ACCORD & ADVANCE & VADT \\
\hline $\begin{array}{l}\text { Major } \\
\text { Endpoints }\end{array}$ & $\begin{array}{l}\text { CV death -or- } \\
\text { Non-fatal MI/stroke }\end{array}$ & $\begin{array}{l}\text { CV death -or- } \\
\text { Non-fatal MI/stroke -or- } \\
\text { macrovacs event }\end{array}$ & $\begin{array}{l}\text { CV death -or- } \\
\text { Non-fatal MI/stroke -or- } \\
\text { macrovacs event }\end{array}$ \\
\hline Study & RCT & RCT & $\mathrm{RCT}$ \\
\hline Design & $\begin{array}{l}\text { Glucose intensive vs. } \\
\text { standard arm, } 2 \times 2 \mathrm{BP} \\
\text { control, +/- fenofibrate } \\
\text { vs. placebo }\end{array}$ & $\begin{array}{l}\text { Glucose intensive vs. } \\
\text { standard arm, } 2 \times 2, \\
\text { +indamine vs. placebo }\end{array}$ & $\begin{array}{l}\text { Glucose intensive vs. } \\
\text { standard arm, } 2 \times 1 \text {, all BP } \\
\text { and lipid } \mathrm{Rx}\end{array}$ \\
\hline
\end{tabular}

Fig. 7. Comparison of the ACCORD, ADVANCE, and VADT studies.

\subsection{The ACCORD study}

In the landmark ACCORD study which revealed surprising results to the medical community, an increase in mortality in the intensively treated diabetes group was observed as compared to the standard therapy group. So adverse was the intensively treated regimen that the study was halted two years early.

The study's authors attributed increased mortality to weight gain, hypoglycemia and possibly specific medications. However, it is reasonable to assume that hypoglycemia was a major contributing factor. In the ACCORD study, the incidence of severe hypoglycemia was significantly higher in the intensively treated group. Over $15 \%$ of patients had severe hypoglycemia as compared to $5 \%$ in the standard treatment group. Increased mortality stemmed from cardiac issues such as cardiac arrhythmias, cardiovascular changes from hyperadrenalism (e.g. heart rate), vascular constriction with its attendant angina, myocardial ischemia and even myocardial infarction, or increased thrombotic tendency with decreased thrombolysis. Regardless of the absolute cause of increased mortality among intensively treated patients, it is clear that intensive treatment, with its extra time, cost, dedication, and more frequent episodes of hypoglycemia and anxiety, did not benefit longterm diabetic patients. 


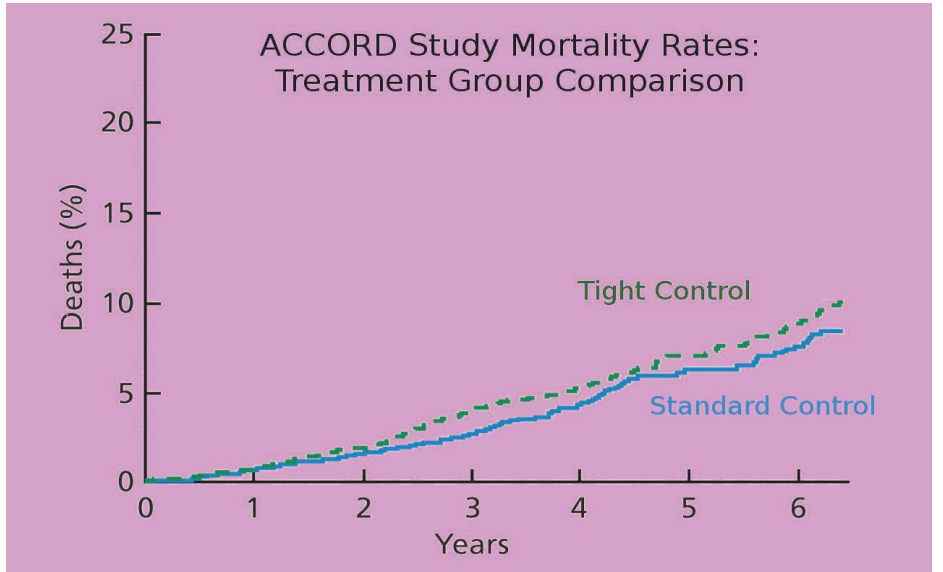

Fig. 8. Mortality rates among treatment regimens in the ACCORD study (adapted from ACCORD Study Group, 2011).

\subsection{The ADVANCE study}

In the ADVANCE study, gliclizide was used in the intensively treated group of type II diabetes patients and compared with standard therapy in over 11,000 patients. Though severe hypoglycemia was infrequent in both arms of the study, nearly twice the proportion of patients in the intensively treated group reported episodes of hypoglycemia $(2.7 \% \mathrm{vs}$. $1.5 \%$ ). The intensity of diabetes control was an independent predictor of microvascular and macrovascular events. There is also a significant correlation between severe hypoglycemia and all-cause mortality. This is true of both non-cardiovascular and cardiovascular mortality. From the results of the ADVANCE study, it can be concluded that hypoglycemia is at least a key marker if not the cause of clinical outcomes.

\subsection{The VADT study}

From the VADT trial, it appears that there can be harm from intense glucose control. This study examined the benefit of tight control in type II diabetics and developed the conclusions that tight control did not lower the risk of cardiovascular events, and that while tight control may be beneficial during the first ten years of diabetes treatment, it may be harmful to elderly patients.

\subsection{The NICE-SUGAR trial}

The NICE-SUGAR trial examined 6,108 patients in the intensive-care unit (NICE-SUGAR Study, 2009). There were two comparable groups who were treated differently, the intensively treated group (goal glucose $81-108 \mathrm{mg} / \mathrm{dl}$ ) and the conventional therapy group (goal glucose glucose $<180 \mathrm{mg} / \mathrm{dL}$ ). A total of 829 patients $(27.5 \%)$ in the intensive-control group and $751(24.9 \%)$ in the conventional-control group died during the study. In the trial there was a significant increase in severe hypoglycemic events observed in the intensively treated group as compared to the conventional therapy group, and an increase in mortality without any clear evidence of health outcome benefit. Indeed, mortality increased in the intensively treated group across all subgroups, including surgical patients. In critically ill 
patients, an association was revealed between even mild or moderate hypoglycemia and mortality. The more severe the hypoglycemia, the greater the risk of death. Again, with the added expense, increased work load on hospital staff and patients, and increased hypoglycemia, it can be concluded that there is no benefit in intensely treating diabetes in enfeebled populations, such as those in the intensive-care unit. Intensive treatment may very well be harmful.

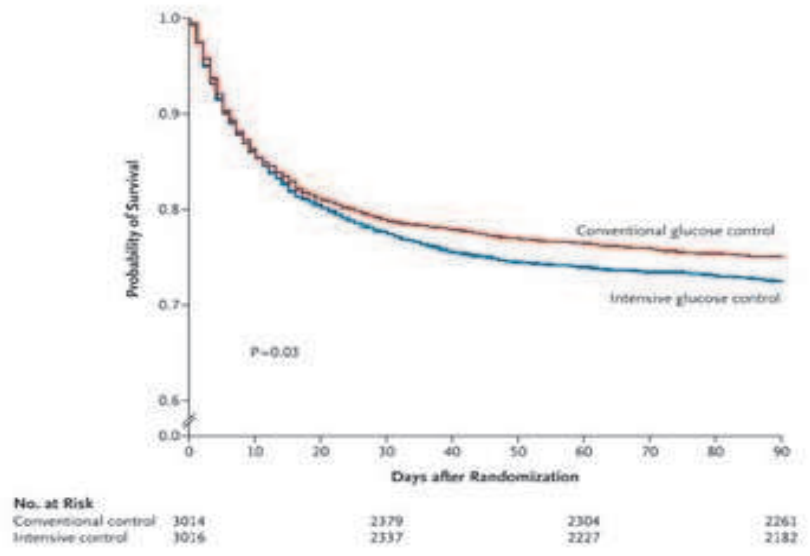

Fig. 9. Comparison of survival rates in the NICE-SUGAR study (adapted from NICESUGAR Study Investigators, 2009).

\section{Hypoglycemia, anxiety and diabetes control}

A non-trivial aspect of treating a diabetic patient with a history of hypoglycemic incidents is the potential resistance of the patient to an aggressive diabetes management plan. It has been shown that more than $54 \%$ of patients with diabetes are anxious about hypoglycemia most of or all of the time (Álvarez et al., 2008; Zhang, 2010). Diabetic patients with episodes of hypoglycemia reported significantly higher rates of shakiness, sweating, excessive fatigue, drowsiness, inability to concentrate, dizziness, hunger, asthenia and headache. These patients tend to allow their average glucose level to become higher, and are resistant to suggestions as to how to improve their diabetes control.

In treating patients clinically, it is clear that the worry of developing hypoglycemia stops many patients from achieving diabetes management goals. The more episodes of hypoglycemia, the lesser the compliance.

\section{Hypoglycemia and health care costs}

Hypoglycemia increases a patient's overall health costs significantly. In a cohort of 2,664 patients evaluated with a health-care claims database, the average annual health-care expenditure for diabetic patients with hypoglycemia was $\$ 3,169$ versus $\$ 1,812$ for diabetics who do not have hypoglycemia (Zhang, 2010). The average usage of short term disability in diabetic patients with hypoglycemia is approximately twice that of patients who do not 
have hypoglycemia (19.5 days annually vs. 11 days annually). In Sweden, a study assessed the burden of hypoglycemia in a 309 patient diabetic population over 35 years of age (Lundkvist et al., 2005). The results showed that 37\% of patients reported symptoms of hypoglycemia during the preceding month. These patients were more affected by their diabetes, reported lower general health, and were more anxious about hypoglycemia than those without hypoglycemia. In this study, the direct and indirect costs of hypoglycemia per patient were estimated to be an additional \$27 per month (in Sweden).

\section{Hypoglycemia prevention}

One antecedent hypoglycemic reaction decreases the epinephrine and symptoms score significantly for at least twenty-four hours. This blunted epinephrine response and hypoglycemic unawareness allows for increased incidences of hypoglycemia. Thus, hypoglycemia begets hypoglycemia, making it imperative for a patient to be particularly vigilant following a hypoglycemic reaction. This is especially important for two days following a hypoglycemic reaction. This entails more blood glucose monitoring and relaxing the blood glucose goal.

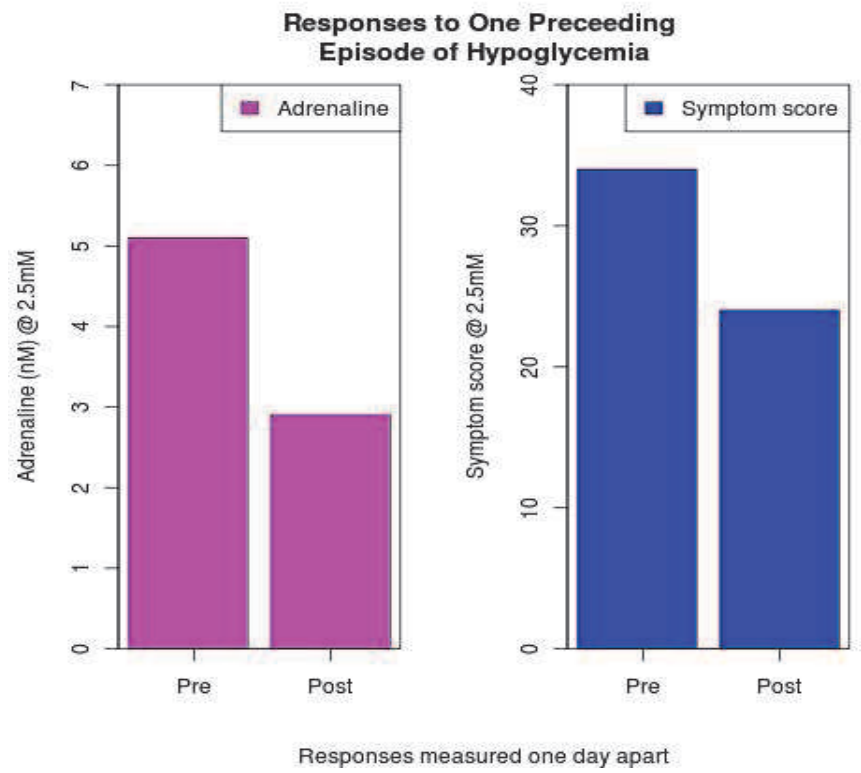

Fig. 10. Epinephrine and symptoms score, pre- and post-hypoglycemia (adapted from Heller, 2010).

For those with hypoglycemic unawareness, the pre-meal glucose level should be no lower than $140 \mathrm{mg} / \mathrm{dL}$. The art of diabetic control comes into play, as the patient must be keenly aware of early signs of hypoglycemia. If hypoglycemia is anticipated, the patient must check his or her blood glucose levels using finger-sticks more often and he or she must try to identify patterns in a monitoring diary to predict potential causes of this insulin side effect. The insulin dose must do be adjusted with consideration of activity and lifestyle. 
Without patient understanding and cooperation, there is little the physician can do to prevent hypoglycemia. Even raising the average glucose goal to very high levels will not suffice without the additional effort of more monitoring on the part of the patient. Training programs exist to help those with hypoglycemic unawareness (blood glucose awareness training, BGAT), and these have been shown to help patients significantly (Cox, 2001).

There is a novel approach of giving an alpha glucosidase inhibitor of the enteric enzyme which slows carbohydrate breakdown and thus prolongs absorption of carbohydrates in order to make glucose available for longer periods. This has been shown to decrease hypoglycemia.

Continuous glucose monitors are a new tool that enhances a patient's ability to achieve this. Currently, several continuous glucose monitoring devices are available (Guardian Realtime, Dexcom Seven Plus, Abbott Freestyle Navigator).

These devices all measure interstitial glucose levels, and though this metric lags whole blood glucose levels by at least 15 minutes, this provides the patient with relatively up-todate information. It should be noted that these devices are designed to be supplemental to finger-stick glucose monitoring (Chico et al., 2003). They are designed to alert the patient if blood glucose levels fall past a pre-set level. These devices are particularly beneficial for patients who have frequent hypoglycemia and/or hypoglycemia unawareness (Beck, 2011). It can also identify dangerously low overnight blood glucose levels, as patients do not monitor their glucose level during this time. The STAR trial has shown that using these machines allows a $1 \%$ decrease in hemoglobin A1c levels without an increase in hypoglycemia.

\subsection{Risks associated with elderly populations}

In elderly diabetic patients who are experiencing episodes of hypoglycemia, the intensity of diabetes treatment should be reduced. A looser blood glucose range is more tolerable than hypoglycemic events, as this population is particularly vulnerable to accidents stemming from low blood sugar. A single hypoglycemic reaction could mean a fall, a broken hip, and a $50 \%$ six month mortality rate corresponding to elderly patients post-fracture.

In addition, elderly populations show a decrease in the counter-regulatory hormonal responses to hypoglycemia, and also a decrease in the symptomatic responses to hypoglycemia. Decreased cognition, renal impairment or polypharmacy are contributing causes. Hypoglycemia should be carefully monitored, as presenting features may be atypical and easily misinterpreted, resulting in delayed treatment. It should be noted that simply raising the hemoglobin A1c target level may not be adequate to prevent hypoglycemia in those with poor glucose control (Munshi, 2011).

\subsection{Prevention in elderly populations}

More frequent glucose monitoring is perhaps the best method for preventing hypoglycemia. With this approach, the elderly patient will be more quickly able to respond to dangerously decreasing blood glucose levels.

As recommended by the by the American Academy of Clinical Endocrinologists, elderly patients should avoid medications that cause hypoglycemia, such as sulfonureas. While these medications have excellent uses in other populations, they are more likely to be hazardous to elderly patients. 


\section{Treatment of hypoglycemia}

During symptoms, whenever possible, a patient's blood glucose level should be checked. If the patient is found to be hypoglycemic, eating or drinking a sugary food is rapidly beneficial. The meal does not have to be large, but should provide a good deal of sugar. Examples are 2-3 glucose tablets, glucose gel, 1/2 cup fruit juice or soft drink, 1 tablespoon table sugar, or 4-6 pieces of hard candy. Following ingestion, the patient's glucose level should be checked within 20 minutes to make sure that glucose is rising. If blood glucose remains low (under $70 \mathrm{mg} / \mathrm{dL}$ ), more sugar should be ingested. For patients with a history of hypoglycemia, a snack or drink containing sugar should be available at all times to take as soon as symptoms appear.

If a patient is found to exhibit hypoglycemia during sleep, decreasing the evening insulin and/or ingesting a complex carbohydrate or protein prior to bedtime should be recommended.

In situations where a hypoglycemic diabetic is non-cooperative or non-responsive, injectable glucagon may be given. The typical dose in a glucagon kit is $1 \mathrm{mg}$, which is sufficient to dose a $200 \mathrm{lb}$ person. A full dose is usually not needed for someone weighing less than 150 pounds, such as a child. Side effects include nausea with an excess dosage, and for most patients a half dosage should be sufficient. If glucose hasn't risen in ten to fifteen minutes after the injection, the other half dose can always be given. This approach works rapidly, usually within 15-20 minutes, by causing a large egress of glucose from the liver.

A classic method for treatment of hypoglycemia is 1 ampule of intravenous $50 \%$ dextrose. The dosage for D50W is one half of the ampule, with verification of IV patency after the half of the ampule is given, and a recheck of blood glucose levels before administering the other half. $50 \%$ dextrose is heavily necrotic due to its hyperosmolarity, and should only be given through a patent IV line. Any infiltration can cause massive tissue necrosis.

\section{Conclusion}

As shown in the United Kingdom Prospective Diabetes Study (UKPDS) (UK Hypoglycaemia Study Group, 2007), patients with type II diabetes who tightly control their glucose levels during the first decade after diagnosis (hemoglobin A1c average of $7 \%$ vs $7.9 \%$ ) exhibited a reduced risk of heart attack and death in subsequent years. This engenders within many patients a comfort with tight glucose control. However, patients who attempt tight control for longer durations of diabetes have significantly increased incidences of hypoglycemia, which increases morbidity and mortality. This is evident in both an outpatient or an inpatient setting. In general, patients with more complications from diabetes, older patients, more frequent episodes of hypoglycemia, and longer durations of diabetes lead to more severe instances of hypoglycemia. There are many possible pathological pathways for hypoglycemia to cause sudden death. Less intensive glucose goals to avoid the possibility of hypoglycemia are imperative to consider in the treatment of diabetics.

\section{References}

ACCORD Study Group. Long-Term Effects of Intensive Glucose Lowering on Cardiovascular Outcomes. N Engl J Med 2011; 364:818-828 
American Diabetes Association. Defining and reporting hypoglycemia in diabetes. Diabetes Care. 2005; 28(5): 1245-1249.

Álvarez, Guisasola F., Tofé, Povedano S., Krishnarajah, G., Lyu, R., Marvos, P., Yin, D. Hypoglycaemic symptoms, treatment satisfaction, adherence and their associations with glycaemic goal in patients with type 2 diabetes mellitus: findings from the Real-Life Effectiveness and Care Patterns of Diabetes Management (RECAP-DM) Study. Diabetes Obes Metab. 2008; 10(suppl 1): 25-32.

Beck, R. Juvenile Diabetes Research Foundation continous glucose monitoring study group: Factors predictive of severe hypoglycemia in type 1 diabetes. Diabetes Care. 2011; 10: 1111.

Ben-Ami, H., Nagachandran, P., Mendelson, A.and Edoute, Y. Drug-induced hypoglycemic coma in 102 diabetic patients. Arch. Intern. Med. 1999. 159:281-284.

Bolli, G. Abnormal glucose counter-regulation in insulin-dependent diabetes mellitus: Interaction of anti-insulin antibodies and impaired glucagon and epinephrine secretion. Diabetes. 1983. Vol. 32, No. 2: 134-141.

Bruce, D.G. Severe hypoglycaemia and cognitive impairment in older patients with diabetes: the Fremantle Diabetes Study. Diabetologia. 2009; 52: 1808-1815.

Chan, S.P., Ji, L.N., Nitiyanant, W., Baik, S.H., Sheu, W.H.H. Hypoglycemic symptoms in patients with type 2 diabetes in Asia-Pacific Real-life effectiveness and Care Patterns of Diabetes Management: the RECAP-DM study. Diabetes Res. Clin. Pract. 2010; 89(2): e30-e32.

Chico, A., Vidal-Ríos, P., Subirà, M., Novials, A. The continuous glucose monitoring system is useful for detecting unrecognized hypoglycemias in patients with type 1 and type 2 diabetes but is not better than frequent capillary glucose measurements for improving metabolic control. Diabetes Care. 2003. 26(4): 1153-1157.

Canadian Diabetes Association. Clinical Practice Guidelines Expert Committee. Hypoglycemia. Can J Diabetes. 2008. 32(suppl 1): S62-S64.

Cox, D. Blood Glucose Awareness Training (BGAT-2): Long-term benefits. Diabetes Care. 2001. Vol. 24, No. 4: 637-642

Cryer, P.E. Mechanisms of sympathoadrenal failure and hypoglycemia in diabetes. J. Clin. Invest. 2006. 116(6): 1470-1473.

Cryer, P.E. Hypoglycemia, functional brain failure, and brain death. J. Clin. Invest.2007. 117(4): 868-870.

Cryer, P.E., Davis, S.N., Shamoon, H. Hypoglycemia in diabetes. Diabetes Care.2003. 26(6): 1902-1912.

Davis, T. Determinants of severe hypoglycemia complicating type 2 diabetes: The Fremantle Diabetes Study. Journal of Clinical Endocrinology and Metabolism. 2009.

Donnelly, L.A. Frequency and predictors of hypoglycaemia in Type 1 and insulin-treated Type 2 diabetes: a population-based study. Diabet. Med. 2005. ;22(6): 749-755.

European Medicines Agency. Note for guidance on clinical investigation of medicinal products in the treatment of diabetes mellitus. Published May 30,2002. http://www.ema.europa.eu/docs/en_GB/document_library/Scientific_guideline /2009/09/WC500003262.pdf. Accessed September 7, 2010.

Heller, Simon. Hypoglycemia in Type II diabetes Module 1 and 2. Ourceatus.org. 2010.

Johnston, S. Evidence linking hypoglycemic events to an increased risk of acute cardiovascular events in patients with type 2 diabetes. Diabetes Care. 2011. 10-15. 
Lee, S. Influence of Autonomic Neuropathy on QTc Interval Lengthening During Hypoglycemia in Type 1 Diabetes. Diabetes. 2004. Vol. 53, No. 6: 535-1542.

Lundkvist, J., Berne, C., Bolinder, B., Jönsson, L. The economic and quality of life impact of hypoglycemia. Eur. J. Health Econom. 2005. 6(3): 197-202.

Matyka, K., Evans, M., Lomas, J., Cranston, I., MacDonald, I., Amiel, S.A. Altered hierarchy of protective responses against severe hypoglycemia in normal aging in healthy men. Diabetes Care. 1997. 20(2): 135-141.

McCrimmon, R., Sherwin, R. Hypoglycemia in Type 1 Diabetes. Diabetes. 2010. 59(10): 23332339.

Meneilly, G.S., Cheung, E., Tuokko, H. Altered responses to hypoglycemia of healthy elderly people. J. Clin. Endocrinol. Metab. 1994. 78(6): 1341-1348.

Munshi, M. Frequent Hypoglycemia Among Elderly Patients With Poor Glycemic Control. Arch. Intern. Med. 2011. 171(4): 363-364.

NICE-SUGAR Study Investigators. Intensive versus Conventional Glucose Control in Critically Ill Patients. N. Engl. J. Med. 2009. 360: 1283-1297.

Piette, J.D., Richardson, C., Valenstein, M. Addressing the Needs of Patients With Multiple Chronic Illnesses: The Case of Diabetes and Depression. The American Journal of Managed Care. 2004. 10:152-162.

Rossetti, P. Prevention of hypoglycemia while achieving good glycemic control in type 1 diabetes: the role of insulin analogs. Diabetes Care. 2008. 31: S113-20.

Spiller, H.A. Unintentional Therapeutic Errors Involving Insulin in the Ambulatory Setting Reported to Poison Centers. The Annals of Pharmacotherapy. 2011. 45(1): 1-6.

UK Hypoglycaemia Study Group. Diabetolgia. 2007. 50: 1140-1147.

Warren, R.E., Hypoglycaemia and cognitive function. Diabetes Obes. Metab. 2005. 7: 493-503.

Yale, J.F., Begg, I., Gerstein, H. Canadian Diabetes Association clinical practice guidelines for the prevention and management of hypoglycemia in diabetes. Can. J. Diabetes. 2001. 26(1): 22-35.

Zhang, Y. The Burden of hypoglycemia in type 2 diabetes: A systemic review of patient and economic perspectives. JCOM Journal. 2010. Vol 17, No. 12: 547-557. 


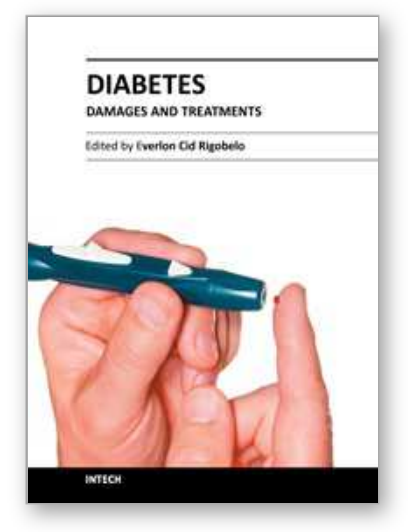

\author{
Diabetes - Damages and Treatments \\ Edited by Prof. Everlon Rigobelo
}

ISBN 978-953-307-652-2

Hard cover, 348 pages

Publisher InTech

Published online 09, November, 2011

Published in print edition November, 2011

Over the last few decades the prevalence of diabetes has dramatically grown in most regions of the world. In 2010, 285 million people were diagnosed with diabetes and it is estimated that the number will increase to 438 million in 2030. Hypoglycemia is a disorder where the glucose serum concentration is usually low. The organism usually keeps the serum glucose concentration in a range of 70 to $110 \mathrm{~mL} / \mathrm{dL}$ of blood. In hypoglycemia the glucose concentration normally remains lower than $50 \mathrm{~mL} / \mathrm{dL}$ of blood. Hopefully, this book will be of help to many scientists, doctors, pharmacists, chemicals, and other experts in a variety of disciplines, both academic and industrial. In addition to supporting researcher and development, this book should be suitable for teaching.

\title{
How to reference
}

In order to correctly reference this scholarly work, feel free to copy and paste the following:

Paul Norwood and Alex Fogel (2011). Diabetes Control and Hypoglycemia, Diabetes - Damages and Treatments, Prof. Everlon Rigobelo (Ed.), ISBN: 978-953-307-652-2, InTech, Available from: http://www.intechopen.com/books/diabetes-damages-and-treatments/diabetes-control-and-hypoglycemia

\section{INTECH}

open science | open minds

\section{InTech Europe}

University Campus STeP Ri Slavka Krautzeka 83/A 51000 Rijeka, Croatia Phone: +385 (51) 770447

Fax: +385 (51) 686166 www.intechopen.com

\section{InTech China}

Unit 405, Office Block, Hotel Equatorial Shanghai No.65, Yan An Road (West), Shanghai, 200040, China 中国上海市延安西路65号上海国际贵都大饭店办公楼405单元 Phone: +86-21-62489820

Fax: $+86-21-62489821$ 
(C) 2011 The Author(s). Licensee IntechOpen. This is an open access article distributed under the terms of the Creative Commons Attribution 3.0 License, which permits unrestricted use, distribution, and reproduction in any medium, provided the original work is properly cited. 